\title{
Transformation of phase space densities under the coordinate changes of accelerator physics
}

\author{
Gabriele Bassi* \\ Department of Physics, University of Liverpool, Liverpool, L69 7ZE, United Kingdom, \\ and The Cockcroft Institute, Daresbury, WA4 4AD, United Kingdom \\ James A. Ellison ${ }^{\dagger}$ and Klaus Heinemann ${ }^{\ddagger}$ \\ Department of Mathematics and Statistics, University of New Mexico, Albuquerque, New Mexico 87131, USA \\ Robert Warnock ${ }^{\S}$ \\ SLAC National Accelerator Laboratory, Stanford University, Menlo Park, California 94025, USA, \\ and Lawrence Berkeley National Laboratory, University of California, Berkeley, California 94720, USA \\ (Received 23 February 2010; published 14 October 2010)

\begin{abstract}
In self-consistent modeling of many-particle systems it is convenient to solve the Maxwell equations for self-fields in coordinates based in the laboratory with time as the evolution variable, but conventional and also convenient to follow particle motion in Frenet-Serret coordinates referred to a reference orbit, with arclength along that orbit as the evolution variable. We refer to these two pictures as the laboratory system and beam system descriptions, while emphasizing that a Lorentz transformation is not involved; it is only a matter of two alternative descriptions of motion in one inertial frame. The problem then arises of how to express the laboratory system charge/current density for the Maxwell equations in terms of the phase space density described in the beam system. We find the exact expression, then make justified approximations to put the formula in a simple and practical form. Incidentally, we derive exact and approximate equations of motion in the different coordinates, without the use of the canonical formalism. The results have been applied in a study of coherent synchrotron radiation in bunch compressors.
\end{abstract}

DOI: 10.1103/PhysRevSTAB.13.104403

\section{INTRODUCTION}

For calculations from first principles in beam dynamics it is useful to view the phase space density, and the corresponding charge/current density, in alternative coordinate systems. This is noticed especially in self-consistent calculations where one computes the fields produced by the beam ("self-fields") and their reciprocal effect on the phase space distribution of the beam itself. The Maxwell equations for the self-fields are best solved in coordinates (Cartesian or otherwise) with a fixed relation to the laboratory inertial frame, with time as the evolution variable. On the other hand, it is conventional and usually convenient to discuss particle motion in coordinates which describe positions in terms of displacements from a reference curve (essentially Frenet-Serret coordinates) and use arclength $s$ on the reference curve rather than time $t$ as the independent variable of the differential equations of motion. A key provision here is that $s$ is a monotonically increasing function of time. We refer to these two descriptions as the "lab system" and the "beam system," while emphasizing that no Lorentz transformation is involved. It

\footnotetext{
*gbassi@bnl.gov

†ellison@math.unm.edu

"heineman@math.unm.edu

§warnock@slac.stanford.edu
}

PACS numbers: 29.27.Bd, 41.75.-i, 52.65.Ff, 02.30.Hq

is merely a matter of two alternative pictures of the motion in one inertial frame.

Dynamics will be studied in terms of the beam system phase space density, say by the Vlasov equation or by a particle model. Then we have the problem of expressing the lab system charge/current density, the source for Maxwell's equations, in terms of that density. This expression will be obtained by finding the transformation (58) of the phase space density under the coordinate change, basically by invoking particle conservation, then doing projections to get the charge/current density. Our method is quite general in the sense that it would apply to any system of ordinary differential equations in which there is a dependent variable which is a monotonic function of the independent variable. Of course, our method can also be applied to the Liouville evolution, i.e., the Vlasov evolution when self-fields are absent.

It turned out that a clear derivation of the transformation densities given by (58) is more subtle than we first expected, mostly because of the change of evolution variable from $t$ to $s$. This change of independent variable is standard and well understood in the context of the differential equations themselves. Furthermore, each differential equation has an associated phase space density; it is the relation between these that, as far as we know, was not well understood. In fact, one of us doubted that such a transformation exists. The discussion of the transformation of densities under the change of evolution variable from $t$ to $s$ is 
discussed in Sec. IV B. This section is essentially selfcontained and so the reader who is only interested in this central part of our paper can focus on the derivation of (40) and (42).

Our intention is to give a thorough derivation, first with exact equations, but finally making justified approximations to put the results in practically useful form. After those approximations the lab frame charge and current densities are given by Eqs. (73) and (74), so simple and appealing that they could almost be guessed at the outset.

As far as we know from a limited view of the literature, the precise problem that we pose and solve has scarcely been recognized heretofore. Nevertheless, at least our approximate result (73) and (74) might somehow be implicit in certain self-consistent computer codes that solve Maxwell's equations in the lab frame.

We find the exact equations of motion, beginning with the Lorentz force, in four different coordinate systems: the lab system (L), beam system 1 (B1), beam system 2 (B2), and the conventional beam system (B). Here B1 uses the Frenet-Serret coordinates $(s, x)$ and retains $u=c t$ as the evolution variable. Then B2 replaces $u$ by $s$ as the evolution variable; thus $u$ becomes a dependent variable in the equations of motion. Finally, we replace the dependent variable $u$ by $z=s-\beta_{r} u$ and introduce the relative momentum deviation $p_{z}=\left(\gamma-\gamma_{r}\right) / \gamma_{r}$ to obtain the usual beam system setup (B). All of this is done by direct algebra without the use of the canonical formalism.

In each of the coordinate systems we present the phase space density, and explore relations between the various densities. Approximating the exact equations of motion in $\mathrm{B}$, we find equations without self-fields as usually stated in the literature. Realizing our main goal, the sources for the self-fields are written in terms of the system B phase space density.

We were led to this study in the course of work on coherent synchrotron radiation, especially for single-pass systems such as bunch compressors, and have applied the present results in that context [1]. Our discussion will sometimes betray its source in the problem of synchrotron radiation, especially in the restriction that only planar particle motion is considered. Believing that the main effect of synchrotron radiation comes from the dominant curvature of a nearly planar orbit, we can postpone refinements coming from motion perpendicular to the plane (vertical motion). Within the planar restriction, the discussion applies as well to multipass systems including storage rings. An extension to allow vertical motion could be made following similar lines of argument, introducing a third Frenet-Serret coordinate to define the extended beam frame.

It should be mentioned that the use of Frenet-Serret coordinates on a reference curve has its limitations. As Forest and others have emphasized, that technique is less useful when guiding fields are complicated, as in fringe fields of magnets and in certain IP regions where fields for detector magnets appear along with normal accelerator magnets. Forest's response is to use local coordinate patches with algorithms for splicing them together [2]. We hope that our general view on transformation of densities would be of value in such a coordinate scheme.

The paper is organized as follows. In Sec. II we state the system L description, including the equations of motion and the phase space density. In Sec. III we introduce Frenet-Serret coordinates, keep $u=c t$ as the evolution variable, and determine the phase space density in B1 in terms of that for $\mathrm{L}$. We also derive the equations of motion in B1. In Sec. IV we define B2 by changing the evolution variable from $u$ to arclength $s$. We then come to the main analytic step of the paper, relating the phase space density in $\mathrm{B} 2$ to that in B1. As mentioned above, this subsection is essentially self-contained. Section V introduces the B coordinates and relates the density in these coordinates to the density in B2. In Sec. VI the main goal of the paper is reached, to write the system $\mathrm{L}$ charge/current density in terms of the system B phase space density. Section VII discusses the initial value problem for the Vlasov equation in B. In a strict sense this problem is ill posed, but to a good approximation it is replaced by a well-posed problem in practice. Section VIII introduces approximations that lead to the practical equations that we have applied in numerical computations; see Refs. [1,3-8].

\section{EQUATIONS OF MOTION IN LAB SYSTEM}

We consider a model where particles undergo planar motion between two infinite, parallel, perfectly conducting plates. The plates, modeling the vacuum chamber, are perpendicular to the $Y$ axis and separated by a distance $h=$ $2 g$. The $Y$ direction is "vertical." We consider the righthanded system $(Z, X, Y)$ and the only external force is due to a magnetic field $B_{\text {ext }}(Z)$ in the $Y$ direction. The general setup for this planar motion is illustrated in Fig. 1. The system $\mathrm{L}$ reference curve will be denoted by $\mathbf{R}_{r}(s)$ and is discussed for the general case in Sec. III A. The reference

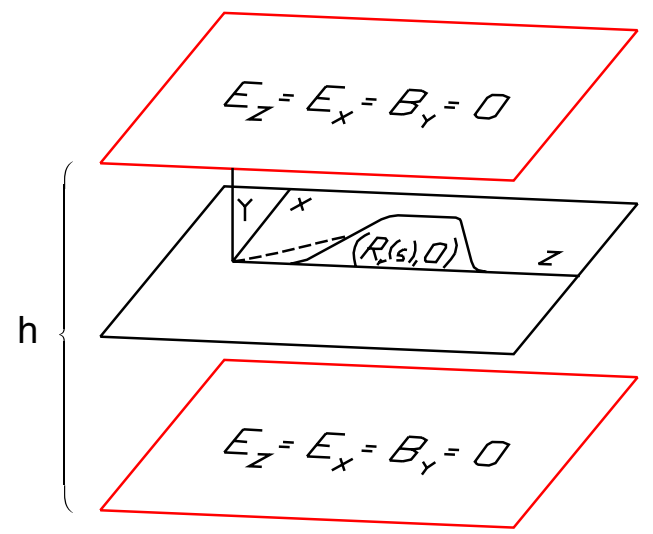

FIG. 1. Basic lab system setup. 
curve illustrated in Fig. 1 is for a four magnet chicane. We suppose that a vertically thin bunch can be modeled by a sheet bunch, i.e., a bunch whose charge density is located in the $Y=0$ plane. Furthermore, for the sheet bunch the only self-field components of consequence are $\mathbf{E}_{\|}=$ $\left(E_{L Z}, E_{L X}\right)$ and $B_{Y}$, where these are $\left(E_{Z}, E_{X}, B_{Y}\right)$ evaluated at $Y=0$. Here we summarize the $\mathrm{L}$ equations of motion, details can be found in Refs. [1,3-8].

With the above setup, the Lorentz equation can be written as

$$
\begin{aligned}
\dot{\mathbf{R}} & =\frac{\mathbf{P}}{m \gamma(\mathbf{P}) c}, \\
\dot{\mathbf{P}} & =\frac{q}{c}\left[\mathbf{E}_{\|}(\mathbf{R}, u)+\frac{B_{\mathrm{ext}}(Z)+B_{Y}(\mathbf{R}, u)}{m \gamma(\mathbf{P})} J \mathbf{P}\right] \\
& =: \frac{q}{c} \mathbf{F}(\mathbf{R}, \mathbf{P}, u),
\end{aligned}
$$

where $m$ and $q$ are the particle mass and charge, $u=c t$, $\cdot=d / d u, \mathbf{R}=(Z, X), \mathbf{P}=\left(P_{Z}, P_{X}\right)$, and

$$
J=\left(\begin{array}{cc}
0 & 1 \\
-1 & 0
\end{array}\right), \quad \gamma(\mathbf{P})=\left[1+\frac{\mathbf{P} \cdot \mathbf{P}}{m^{2} c^{2}}\right]^{1 / 2} .
$$

The self-field components of relevance, with the boundary condition for parallel plates, are given by

$$
\begin{aligned}
\mathcal{F}_{L}(\mathbf{R}, u)= & \left(\mathbf{E}_{\|}, B_{Y}\right)(\mathbf{R}, u) \\
= & -\sum_{k=-\infty}^{\infty}(-1)^{k} \int_{\mathbb{R}^{2}} d \mathbf{R}^{\prime} \\
& \times \frac{\mathbf{S}\left(\mathbf{R}^{\prime}, u-\left[\left|\mathbf{R}^{\prime}-\mathbf{R}\right|^{2}+(k h)^{2}\right]^{1 / 2}\right)}{4 \pi\left[\left|\mathbf{R}^{\prime}-\mathbf{R}\right|^{2}+(k h)^{2}\right]^{1 / 2}} .
\end{aligned}
$$

This is obtained as a solution of the wave equation with inhomogeneous term $\delta(Y) \mathbf{S}(\mathbf{R}, u)$, where

$$
\mathbf{S}(\mathbf{R}, u)=Z_{0}\left(\begin{array}{c}
c \partial_{Z} \rho_{L}+\partial_{u} J_{L, Z} \\
c \partial_{X} \rho_{L}+\partial_{u} J_{L, X} \\
\left(\partial_{X} J_{L, Z}-\partial_{Z} J_{L, X}\right) / c
\end{array}\right) .
$$

Here $\mathbf{J}_{L}=\left(J_{L, Z}, J_{L, X}\right)$ and $Z_{0}=\left(\mu_{0} / \epsilon_{0}\right)^{1 / 2}$ is the impedance of free space, where $\mu_{0}$ and $\epsilon_{0}$ are the free space magnetic permeability and electric permittivity, respectively. Without the parallel plate boundary condition only the $k=0$ term remains. The derivation of (3) is in [1].

The 2D charge and current densities are given by

$$
\begin{gathered}
\rho_{L}(\mathbf{R} ; u)=Q \int_{\mathbb{R}^{2}} d \mathbf{P} f_{L}(\mathbf{R}, \mathbf{P} ; u), \\
\mathbf{J}_{L}(\mathbf{R} ; u)=Q \int_{\mathbb{R}^{2}} d \mathbf{P}[\mathbf{P} / m \gamma(\mathbf{P})] f_{L}(\mathbf{R}, \mathbf{P} ; u) .
\end{gathered}
$$

Here $Q=N q$ is the total charge and $N$ is the number of particles. The system $L$ phase space density is denoted by $f_{L}=f_{L}(W ; u)$, where $W=\left(Z, X, P_{Z}, P_{X}\right)$. We take $\int_{\mathbb{R}^{4}} d W f_{L}=1$, so that $\int_{A} d W f_{L}(W ; u)$ is the fraction of the bunch in $A$. This pattern will be followed for all other phase space densities; they will be normalized to 1 , and the density variables will be separated from the evolution variable by a semicolon. Strictly speaking, a density will be a family of densities parametrized by an evolution variable; however, we assume this will be clear to the reader.

We write (1) in the compact form

$$
\dot{W}=F(W, u) .
$$

The evolution law for $f_{L}$ is the Vlasov equation,

$$
\partial_{u} f_{L}+\nabla_{W} \cdot\left[F(W, u) f_{L}\right]=0
$$

which can also be viewed as a particle conservation law in this mean field approach. We note that $F$ depends implicitly on $f_{L}$. It is easily checked that $F(\cdot, u)$ is divergence free, i.e., $\nabla_{W} \cdot F(W, u)=0$, so that (8) becomes

$$
\partial_{u} f_{L}+F(W, u) \cdot \nabla_{W} f_{L}=0 .
$$

\section{BEAM SYSTEM 1}

\section{A. The system $L$ reference curve}

The system $\mathrm{L}$ reference curve $\mathbf{R}_{r}(s)=\left[Z_{r}(s), X_{r}(s)\right]^{T}$ is parameterized by arclength $s$ and is defined by the initial value problem,

$$
\begin{aligned}
& \mathbf{R}_{r}^{\prime}=\mathbf{t}, \quad \mathbf{t}^{\prime}=\frac{q B_{\mathrm{ext}}\left(Z_{r}\right)}{P_{r}} J \mathbf{t}, \\
& \mathbf{R}_{r}(0)=0, \quad \mathbf{t}(0)=(1,0) .
\end{aligned}
$$

Here $^{\prime}=d / d s$ and the unit tangent vector along the reference curve is denoted by $\mathbf{t}(s)$. The reference particle is defined in the lab system by $\mathbf{R}(u)=\mathbf{R}_{r}\left(\beta_{r} u\right), \mathbf{P}(u)=$ $P_{r} \mathbf{t}\left(\beta_{r} u\right)$, where $\beta_{r}=|\dot{\mathbf{R}}(u)|$ is the constant speed of the reference particle, $P_{r}=m \gamma_{r} \beta_{r} c$, and $\gamma_{r}=\left(1-\beta_{r}^{2}\right)^{-1 / 2}$. Note that the reference particle solves (1) without the selffields. In the following we assume that $B_{\text {ext }}$ is smooth so that $\mathbf{R}_{r}(s)$ is smooth. In Sec. III B, we will add one more assumption on $\mathbf{R}_{r}$ so that all our mathematical operations to follow are legitimate. In applications one often assumes hard edge magnets, and then more care must be taken. In that case $B_{\text {ext }}$ is piecewise smooth with jumps and $\mathbf{t}(s)$ is $C^{0}$ but not $C^{1}$.

It is necessary to introduce a unit normal vector, $\mathbf{n}$, to the reference curve and we choose $\mathbf{n}(s):=-J \mathbf{t}(s)$, so that $\mathbf{n}$ is a $\pi / 2$ counterclockwise rotation of $\mathbf{t}$. We define the curvature $\kappa$ by $\mathbf{t}^{\prime}=:-\kappa(s) \mathbf{n}$, thus the second equality in (10) gives

$$
\kappa(s)=\frac{q B_{\text {ext }}\left[Z_{r}(s)\right]}{P_{r}},
$$

which is determined once (10) and (11) has been solved. Multiplication of the second equality in (10) by $J$ gives $\mathbf{n}^{\prime}=\kappa(s)$ t. The situation is illustrated in Fig. 2. 


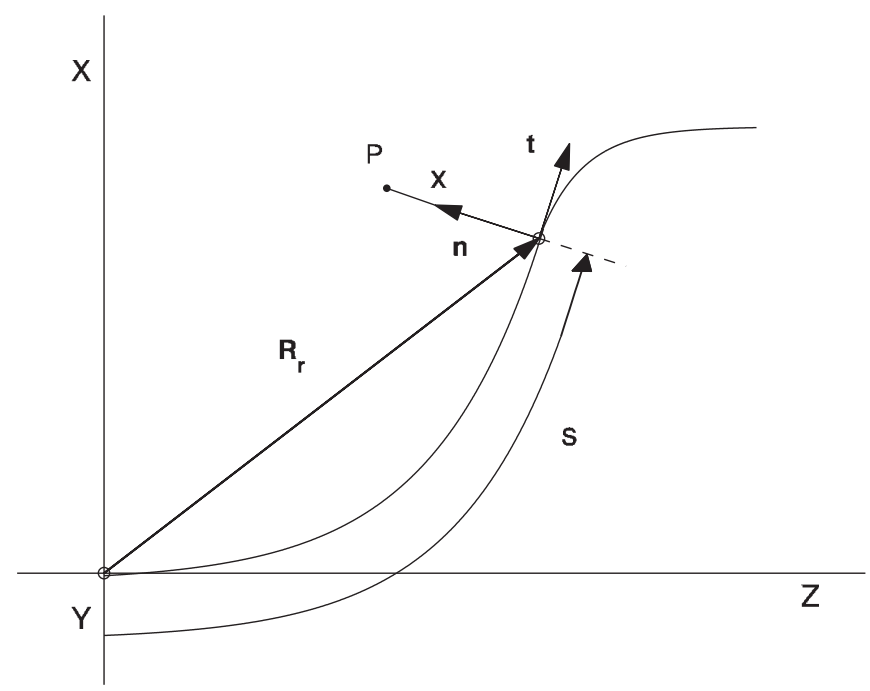

FIG. 2. Beam frame coordinates.

The system $L$ reference curve for a four dipole magnetic chicane is illustrated in Fig. 1. A typical curvature in a dipole of a bunch compressor is of the order of inverse meters so that the radius of curvature is much greater than the dimensions of a typical bunch.

\section{B. Definition of beam system 1}

The Frenet-Serret spatial coordinates are $(s, x)$, where $s$ is the arclength along the reference curve and $x$ is the perpendicular distance along $\mathbf{n}$. Consider the map T: $U \rightarrow$ $\mathbb{R}^{2}$ defined by $\mathbf{T}(s, x)=\mathbf{R}_{r}(s)+x \mathbf{n}(s)$, with domain $U=$ $\left\{(s, x):|x|<x_{M}\right\}$. Since $B_{\text {ext }}$ is smooth, the derivative (Jacobian matrix) exists and is given by $D \mathbf{T}(s, x)=[\{1+$ $\kappa(s) x\} \mathbf{t}(s), \mathbf{n}(s)$. We assume that $\mathbf{R}_{r}$ is such that a positive $x_{M}$ exists so that $\mathbf{T}$ is one-to-one and the derivative is invertible in $U$. The derivative is invertible if $x_{M} \leq$ $\left[\sup _{s \in \mathbb{R}}|\kappa(s)|\right]^{-1}$, since the Jacobian $1+\kappa(s) x$ is then positive in $U$. At this point, we have that $\mathbf{T}: U \rightarrow \mathbf{T}(U)$ is a differentiable bijection with invertible derivative. By the inverse function theorem the inverse is differentiable and $\mathbf{T}(U)$ is open, thus $\mathbf{T}$ is a diffeomorphism. We write the inverse $\mathbf{T}^{-1}: \mathbf{T}(U) \rightarrow U$ as

$$
\mathbf{T}^{-1}(\mathbf{R})=[\hat{s}(\mathbf{R}), \hat{x}(\mathbf{R})]^{T} .
$$

Figure 2 illustrates the transformation $\mathbf{T}$. Clearly $s$ and $x$ determine $\mathbf{R}$. Conversely, given an $\mathbf{R} \in \mathbf{T}(U)$ there is a unique perpendicular line from $\mathbf{R}$ to the reference curve with $|x|<x_{M}$. Thus $s, x, \mathbf{t}(s)$, and $\mathbf{n}(s)$ are determined. For typical bend radii of order meters, $x_{M}$ can be much larger than a typical transverse bunch size.

We assume that the bunch stays close to the reference curve, and that its charge density, $\rho_{L}$, has compact support [it vanishes outside a bounded set within $T(U)$ ], for each $u$. In our numerical computations, we often use a Gaussian initial density which does not exactly satisfy this requirement, but does to a good approximation.
The B1 momentum coordinates $\left(p_{s}, p_{x}\right)$ are defined by $\mathbf{P}=: P_{r}\left[p_{s} \mathbf{t}(s)+p_{x} \mathbf{n}(s)\right]$, where we require $p_{s}$ to be positive. Let $\xi=\left(s, x, p_{s}, p_{x}\right)$ then the phase space transformation $\chi: S_{1} \rightarrow \mathbb{R}^{4}$ is defined by $(\mathbf{R}, \mathbf{P})=\chi(\xi)$, where

$$
\mathbf{R}=\mathbf{R}_{r}(s)+x \mathbf{n}(s), \quad \mathbf{P}=P_{r}\left[p_{s} \mathbf{t}(s)+p_{x} \mathbf{n}(s)\right],
$$

and where the $\mathrm{B} 1$ phase space is

$$
S_{1}=\left\{\left(s, x, p_{s}, p_{x}\right):|x|<x_{M}, p_{s}>0\right\} .
$$

This is also a diffeomorphism and the inverse, $\chi^{-1}: \chi\left(S_{1}\right) \rightarrow S_{1}$ is

$$
\begin{array}{ll}
s=\hat{s}(\mathbf{R}), & p_{s}=\mathbf{P} \cdot \mathbf{t}[\hat{s}(\mathbf{R})] / P_{r}, \\
x=\hat{x}(\mathbf{R}), & p_{x}=\mathbf{P} \cdot \mathbf{n}[\hat{s}(\mathbf{R})] / P_{r} .
\end{array}
$$

We require the support of the system $L$ phase space density $f_{L}(\cdot ; u)$ to be a bounded subset of $\chi\left(S_{1}\right)$ for each $u$ of interest. We also require $p_{s}$ to be positive so that $s$ is a monotonically increasing function of $u$ for each particle in the bunch.

We will need an approximation of the inverse functions $(\hat{s}, \hat{x})$ in a neighborhood of $\mathbf{R}=\mathbf{R}_{r}(y)$. To expand through second order, we apply

$$
f(\mathbf{R}) \approx f\left[\mathbf{R}_{r}(y)\right]+\nabla f\left[\mathbf{R}_{r}(y)\right] \cdot \mathbf{v}+\frac{1}{2} \mathbf{v}^{T} H\left[\mathbf{R}_{r}(y)\right] \mathbf{v},
$$

to both $\hat{s}$ and $\hat{x}$. In (17), $f$ is a scalar, $\mathbf{v}=\mathbf{R}-\mathbf{R}_{r}(y)$ and $H$ is the Hessian matrix of second order partial derivatives. From the identity $\mathbf{R}=\mathbf{R}_{r}[\hat{s}(\mathbf{R})]+\hat{x}(\mathbf{R}) \mathbf{n}[\hat{s}(\mathbf{R})]$ we obtain

$$
\hat{s}\left[\mathbf{R}_{r}(y)\right]=y, \quad \hat{x}\left[\mathbf{R}_{r}(y)\right]=0,
$$

and by differentiation

$$
\begin{aligned}
\nabla \hat{s}(\mathbf{R}) & =\{1+\kappa[\hat{s}(\mathbf{R})] \hat{x}(\mathbf{R})\}^{-1} \mathbf{t}[\hat{s}(\mathbf{R})], \\
\nabla \hat{x}(\mathbf{R}) & =\mathbf{n}[\hat{s}(\mathbf{R})] .
\end{aligned}
$$

From (18) and (19) we obtain $\nabla \hat{s}\left[\mathbf{R}_{r}(y)\right]=\mathbf{t}(y)$, $\nabla \hat{x}\left[\mathbf{R}_{r}(y)\right]=\mathbf{n}(y)$. Differentiating (19) to obtain the second order partial derivatives we find the Hessian matrices for $\hat{s}$ and $\hat{x}$ to be $-\kappa(y)\left[\mathbf{t}(y) \mathbf{n}(y)^{T}+\mathbf{n}(y) \mathbf{t}(y)^{T}\right]$ and $\kappa(y) \mathbf{t}(y) \mathbf{t}(y)^{T}$, respectively. It follows that

$$
\begin{aligned}
& \hat{s}(\mathbf{R}) \approx y+\mathbf{t}(y) \cdot \mathbf{v}-\kappa(y)[\mathbf{t}(y) \cdot \mathbf{v}][\mathbf{n}(y) \cdot \mathbf{v}], \\
& \hat{x}(\mathbf{R}) \approx \mathbf{n}(y) \cdot \mathbf{v}+\frac{1}{2} \kappa(y)[\mathbf{t}(y) \cdot \mathbf{v}]^{2},
\end{aligned}
$$

thus

$$
\begin{aligned}
\left(\begin{array}{c}
\hat{s}(\mathbf{R})-y \\
\hat{x}(\mathbf{R})
\end{array}\right) & =M^{T}(y)\left[\mathbf{R}-\mathbf{R}_{r}(y)\right]+\mathcal{O}\left[\kappa\left\|\mathbf{R}-\mathbf{R}_{r}\right\|^{2}\right], \\
M(y) & :=[\mathbf{t}(y), \mathbf{n}(y)],
\end{aligned}
$$

and dropping the error term we obtain the approximate inverse we seek. In a more detailed analysis we can prove 


$$
\begin{array}{r}
\left\|\hat{s}(\mathbf{R})-y-\mathbf{t}(y) \cdot\left[\mathbf{R}-\mathbf{R}_{r}(y)\right]\right\| \leq L_{\hat{s}}\left\|\mathbf{R}-\mathbf{R}_{r}(y)\right\|^{2}, \\
\left\|\hat{x}(\mathbf{R})-\mathbf{n}(y) \cdot\left[\mathbf{R}-\mathbf{R}_{r}(y)\right]\right\| \leq L_{\hat{x}}\left\|\mathbf{R}-\mathbf{R}_{r}(y)\right\|^{2},
\end{array}
$$

where $L_{\hat{s}}$ and $L_{\hat{x}}$ are $\mathcal{O}(\kappa)$.

The above results hold for $\mathbf{R} \in \mathbf{T}(U)$. In practice, given an $\mathbf{R}$ one must determine if it is in $\mathbf{T}(U)$; we do not address this here. In principle, $\mathbf{T}(U)$ might not be big enough to contain the bunch. In fact, there are reference curves for which $x_{M}$ is smaller than the transverse beam size, thus $x_{M}$ might not exist. Nevertheless, this situation does not occur in practice, e.g., in a four dipole chicane.

\section{Beam system 1 phase space density}

Densities transform according to the Jacobian of the transformation. Let $g_{A}$ be a density in $\mathbb{R}^{n}$ with coordinates $a$ and let $a=\mathcal{T}(b)$ be a coordinate transformation and $\mathcal{J}_{\mathcal{T}}$ the associated Jacobian, then the density in the $b$ coordinates is

$$
g_{B}(b)=g_{A}[\mathcal{T}(b)]\left|\mathcal{J}_{\mathcal{T}}(b)\right|, \quad \mathcal{J}_{\mathcal{T}}(b)=\operatorname{det} D \mathcal{T}(b) .
$$

This transformation law is a simple consequence of particle conservation and the change of variable theorem for integrals; it is also the transformation law for probability densities associated with random variables, $A$ and $B=$ $\mathcal{I}(A)$. It follows that the relation between the B1 phase space density $f_{1}=f_{1}(\cdot ; u)$ and the $\mathrm{L}$ phase space density, $f_{L}(\cdot ; u)$, is

$$
f_{1}(\xi ; u)=f_{L}[\chi(\xi) ; u] P_{r}^{2}[1+\kappa(s) x] .
$$

Since the support of $f_{L}(\cdot ; u)$ is a compact subset of $\chi\left(S_{1}\right)$, the support of $f_{1}(\cdot ; u)$ in (24) is a compact subset of $S_{1}$ and we take the domains of $f_{L}(\cdot ; u)$ and $f_{1}(\cdot ; u)$ to be $\chi\left(S_{1}\right)$ and $S_{1}$, respectively.

\section{Beam system 1 equations of motion}

We are now in a position to derive the B1 equations of motion for $\left(s, x, p_{s}, p_{x}\right)$. We emphasize that $u$ is the independent variable. Differentiating (14) with respect to $u$ and using (1), we obtain

$$
\begin{aligned}
& {[1+\kappa(s) x] \dot{s} \mathbf{t}(s)+\dot{x} \mathbf{n}(s)=} \\
& {\left[P_{r} / m c \gamma(\mathbf{P})\right]\left[p_{s} \mathbf{t}(s)+p_{x} \mathbf{n}(s)\right],} \\
& \left(\dot{p}_{s}+\kappa \dot{s} p_{x}\right) \mathbf{t}(s)+\left(\dot{p}_{x}-\kappa \dot{s} p_{s}\right) \mathbf{n}(s)= \\
& q \mathbf{F} / P_{r} c .
\end{aligned}
$$

Solving (25) and (26) for $\dot{s}, \dot{x}, \dot{p}_{s}, \dot{p}_{x}$, we obtain the equations of motion:

$$
\begin{aligned}
\dot{s} & =\frac{P_{r} / m c}{\gamma\left(p_{s}, p_{x}\right)} \frac{1}{1+\kappa(s) x} p_{s}, \\
\dot{x} & =\frac{P_{r} / m c}{\gamma\left(p_{s}, p_{x}\right)} p_{x}, \\
\dot{p}_{s} & =-\kappa(s) \dot{s} p_{x}+\frac{q}{P_{r} c} \mathbf{t}(s) \cdot \mathbf{F}[\chi(\xi), u], \\
\dot{p}_{x} & =\kappa(s) \dot{s} p_{s}+\frac{q}{P_{r} c} \mathbf{n}(s) \cdot \mathbf{F}[\chi(\xi), u],
\end{aligned}
$$

where

$$
\gamma\left(p_{s}, p_{x}\right)=\left[1+\left(\gamma_{r}^{2}-1\right)\left(p_{s}^{2}+p_{x}^{2}\right)\right]^{1 / 2} .
$$

Without the self-fields

$$
\begin{aligned}
\mathbf{t}(s) \cdot \mathbf{F}[\chi(\xi), u]= & \left\{B_{\mathrm{ext}}\left[Z_{r}(s)-x X_{r}^{\prime}(s)\right] P_{r} / m \gamma\left(p_{s}, p_{x}\right)\right\} p_{x} \\
\mathbf{n}(s) \cdot \mathbf{F}[\chi(\xi), u]= & -\left\{B_{\mathrm{ext}}\left[Z_{r}(s)-x X_{r}^{\prime}(s)\right]\right. \\
& \left.\times P_{r} / m \gamma\left(p_{s}, p_{x}\right)\right\} p_{s}
\end{aligned}
$$

where we have used the fact that $Z(u)=Z_{r}[s(u)]-$ $x(u) X_{r}^{\prime}[s(u)]$, which follows from (14).

The reference particle in $\mathrm{B} 1$ is given by $s(u)=\beta_{r} u$, $p_{s}(u)=1, x(u)=0, p_{x}(u)=0$, which in light of (12) and (29), solves (27) with zero self-fields as it must.

The B1 equations of motion (27) and the density transformation (24) are both results of the phase space transformation (14). So it should come as no surprise that $f_{1}$ as given in (24) solves the B1 Vlasov equation. To be more explicit, we write (27) as

$$
\dot{\xi}=G(\xi, u), \quad \xi=\left(s, x, p_{s}, p_{x}\right) .
$$

We denote the general solution of the B1 equation of motion (30) by

$$
\xi=\varphi\left(u, u_{0} \mid \xi_{0}\right), \quad \text { where } \xi_{0}=\varphi\left(u_{0}, u_{0} \mid \xi_{0}\right) .
$$

The vertical line is meant to emphasize that, for fixed $\left(u, u_{0}\right), \varphi\left(u, u_{0} \mid \cdot\right)$ is a phase space map. By uniqueness the first equality can be inverted to give $\xi_{0}=\varphi\left(u_{0}, u \mid \xi\right)$.

The Vlasov equation associated with (30) is

$$
\partial_{u} f_{1}+\nabla_{\xi} \cdot\left[G(\xi, u) f_{1}\right]=0,
$$

which is equivalent to

$$
\partial_{u} f_{1}+G(\xi, u) \cdot \nabla_{\xi} f_{1}=-\left(\nabla_{\xi} \cdot G\right)(\xi, u) f_{1} .
$$

It can be checked that $f_{1}$ as given in (24) is a solution of (32) given that $f_{L}$ satisfies (8). Also, even though $F$ is divergence free, $G$ is not.

\section{BEAM SYSTEM 2}

Here we change the independent variable from time $u$ to arclength $s$. For this purpose we write (30), and equivalently (27), as

$$
\dot{s}=g_{1}(s, w)
$$




$$
\dot{w}=g_{2}(s, w, u),
$$

where $w:=\left(x, p_{s}, p_{x}\right)$.

\section{A. Definition of system B2 and equations of motion}

From (15) and (27) $\dot{s}$ is positive, thus $s$ can be used as an independent variable in the equations of motion. This allows the definition of the B2 equations of motion as follows. If $\xi(u)=[s(u), w(u)]$ satisfies (33) and (34) then $[u(s), \hat{w}(s)]$, with $\hat{w}(s)=w[u(s)]$, satisfies

$$
\begin{gathered}
u^{\prime}=1 / g_{1}(s, \hat{w})=: h_{1}(\hat{w}, s), \\
\hat{w}^{\prime}=g_{2}(s, \hat{w}, u) / g_{1}(s, \hat{w})=: h_{2}(u, \hat{w}, s),
\end{gathered}
$$

where the function $u(\cdot)$ is the inverse of the function $s(\cdot)$. More compactly we write

$$
\eta^{\prime}=H(\eta, s)
$$

where $\eta=(u, \hat{w}),{ }^{\prime}=d / d s$ and $H=\left(h_{1}, h_{2}\right)$. The reference particle in $\mathrm{B} 2$ is given by $u(s)=s / \beta_{r}, \hat{w}(s)=$ $(0,1,0)$.

For future reference, it is convenient to have the B2 equations of motion in component form; they are

$$
\begin{aligned}
u^{\prime} & =\frac{m c \gamma\left(p_{s}, p_{x}\right)}{P_{r}} \frac{1+\kappa(s) x}{p_{s}}, \\
x^{\prime} & =[1+\kappa(s) x] \frac{p_{x}}{p_{s}}, \\
p_{s}^{\prime} & =-\kappa(s) p_{x}+\frac{q}{P_{r} c} u^{\prime} \mathbf{t}(s) \cdot \mathbf{F}[\chi(\xi), u], \\
p_{x}^{\prime} & =\kappa(s) p_{s}+\frac{q}{P_{r} c} u^{\prime} \mathbf{n}(s) \cdot \mathbf{F}[\chi(\xi), u] .
\end{aligned}
$$

The B2 phase space equivalent to $S_{1}$ is

$$
S_{2}=\left\{\left(u, x, p_{s}, p_{x}\right):|x|<x_{M}, p_{s}>0\right\} .
$$

This is the same subset of $\mathbb{R}^{4}$, although the first components have different meanings.

\section{B. Beam system 2 phase space density}

Here we come to the main analytic step in the paper, relating the $\mathrm{B} 1$ and $\mathrm{B} 2$ phase space densities, $f_{1}$ and $f_{2}$. Consider the set $\left\{(s, u, w):|x|<x_{M}, p_{s}>0\right\}$, where the bunch is confined to a tube around the reference particle $s=\beta_{r} u, w=(0,1,0)$. We want to relate the densities on two arbitrary planes in this space, say $u=u_{1}$ and $s=s_{0}$. We know the B1 phase space density, $f_{1}\left(s, w ; u_{1}\right)$, as a function of $(s, w)$ and we want to derive the system B2 phase space density $f_{2}\left(u, w ; s_{0}\right)$ as a function of $(u, w)$.

Pick a point $\left(s_{1}, w_{1}\right)$ on the $u=u_{1}$ plane, in the support of $f_{1}\left(\cdot ; u_{1}\right)$. By assumption $\left(s_{1}, x_{1}\right) \in U$. Since $g_{1}$ is positive there is a unique point $\left(u_{0}, w_{0}\right)$ on the $s=s_{0}$ plane such that $\left(s_{0}, x_{0}\right)$ is in $U$ and $\left(s_{0}, w_{0}\right)=\varphi\left(u_{0}, u_{1} \mid s_{1}, w_{1}\right)$. Inverting gives

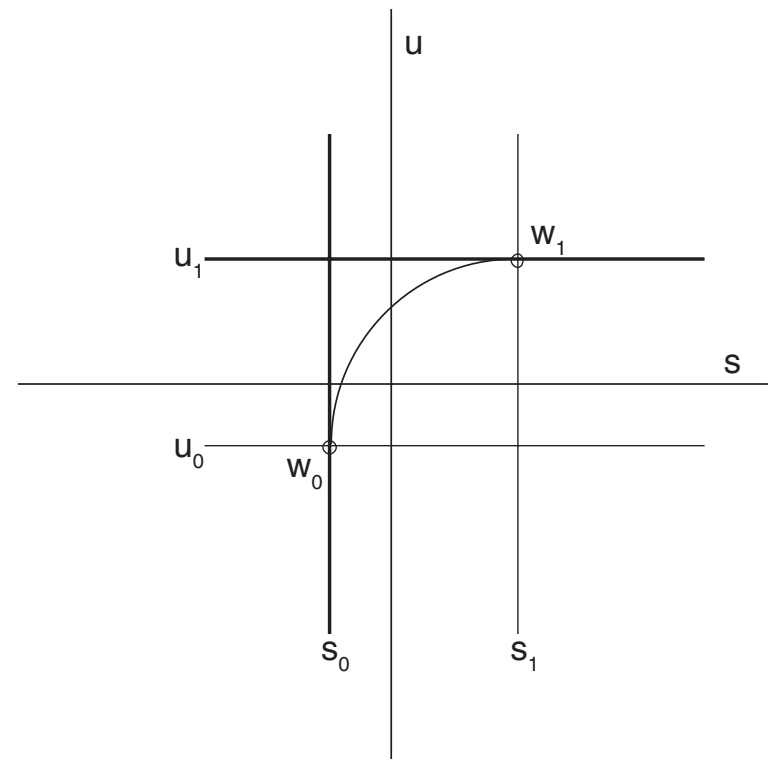

FIG. 3. Relation of $\mathrm{B} 1$ and $\mathrm{B} 2$ densities. The $\mathrm{B} 1$ density on plane $u=u_{1}$ in extended phase space is related to the B2 density on $s=s_{0}$ by the evolution under $\varphi$, represented as a curve connecting $\left(s_{0}, u_{0}, w_{0}\right)$ to $\left(s_{1}, u_{1}, w_{1}\right)$. Think of the $w$ dimension as perpendicular to the $(s, u)$ plane.

$$
\left(s_{1}, w_{1}\right)=\varphi\left(u_{1}, u_{0} \mid s_{0}, w_{0}\right)=: \psi\left(u_{0}, w_{0} ; u_{1}, s_{0}\right) .
$$

The situation for $s_{0}<s_{1}$ is illustrated in Fig. 3. Every point in the support of $f_{1}\left(\cdot ; u_{1}\right)$ is accounted for, thus conservation of particles can be applied in terms of the associated map $\psi:\left(u_{0}, w_{0}\right) \in S_{2} \mapsto\left(s_{1}, w_{1}\right) \in S_{1}$, which depends on the parameters $\left(u_{1}, s_{0}\right)$ that specified the two planes. Applying the general law (23) for transforming densities, we can then state that

$f_{2}\left(u_{0}, w_{0} ; s_{0}\right)=f_{1}\left[\varphi\left(u_{1}, u_{0} \mid s_{0}, w_{0}\right) ; u_{1}\right]\left|\mathcal{J}_{\psi}\left(u_{0}, w_{0} ; u_{1}, s_{0}\right)\right|$.

The Jacobian, calculated in the Appendix, is

$$
\begin{aligned}
& \mathcal{J}_{\psi}\left(u_{0}, w_{0} ; u_{1}, s_{0}\right) \\
& =\operatorname{det}\left[D_{1} \psi, D_{2} \psi\right]\left(u_{0}, w_{0} ; u_{1}, s_{0}\right) \\
& =-g_{1}\left(s_{0}, w_{0}\right) \exp \int_{u_{0}}^{u_{1}} \operatorname{Tr} D_{1} G\left[\varphi\left(u, u_{0} \mid s_{0}, w_{0}\right), u\right] d u,
\end{aligned}
$$

where $g_{1}$ is defined by (33), and $\operatorname{Tr}$ denotes the trace of the matrix that follows.

Now (40) relates densities on the two planes in extended phase space. Replacing $u_{1}$ by $u_{0}$ in (40) gives our principal result:

$$
f_{2}(u, w ; s)=f_{1}(s, w ; u) g_{1}(s, w),
$$

where we have dropped the subscripts. It is this equation that we use in our applications. Since the support of $f_{1}(\cdot ; u)$ is a compact subset of $S_{1}$, the support of $f_{2}(\cdot ; s)$ is a compact subset of $S_{2}$. We have considered smooth den- 
sities. However, in the point particle case, where the densities are delta functions, formula (42) can be derived using the rule for transforming delta functions. It follows that Klimontovich densities obey (42).

Some discussion of the two formulas is worthwhile. The relation (40) shows that, for each $s_{0}, f_{2}\left(\cdot ; s_{0}\right)$ is determined by $f_{1}\left(\cdot ; u_{1}\right)$ at any $u_{1}$ and knowledge of the $\mathrm{B} 1$ flow. The relation (42) shows that at each point $(s, w, u)$ of extended phase space there are two densities depending on whether $(s, w)$ or $(u, w)$ are viewed as the density variables. We derived (42) from (40), but it is interesting to note that the two are equivalent. To derive (40) from (42) we replace $f_{1}$ in (42) by the solution of the Vlasov equation, (32), with initial condition at $u=u_{1}$. Using the method of characteristics we obtain

$$
\begin{aligned}
f_{1}(s, w ; u)= & f_{1}\left[\varphi\left(u_{1}, u \mid s, w\right) ; u_{1}\right] \\
& \times \exp \left[-\int_{u_{1}}^{u} d \tau \operatorname{Tr} D_{1} G[\varphi(\tau, u \mid s, w), \tau]\right] .
\end{aligned}
$$

Replacing $(s, w, u)$ by $\left(s_{0}, w_{0}, u_{0}\right)$ in (42) and (43) we obtain (40).

Instead of evaluating the Jacobian determinant at $u_{1}=$ $u_{0}$ by means of the exact formula (41) we can find the result more simply by a Taylor expansion, which in fact gives added insight. Recalling (30), we expand (39) for $u_{1}$ near $u_{0}$ to obtain

$$
\left(\begin{array}{c}
s_{1} \\
w_{1}
\end{array}\right)=\left(\begin{array}{c}
s_{0} \\
w_{0}
\end{array}\right)+G\left(\xi_{0}, u_{0}\right)\left(u_{1}-u_{0}\right)+\mathcal{O}\left[\left(u_{1}-u_{0}\right)^{2}\right],
$$

whence

$$
\begin{aligned}
& \frac{\partial}{\partial u_{0}}\left(\begin{array}{c}
s_{1} \\
w_{1}
\end{array}\right)=-G\left(\xi_{0}, u_{0}\right)+\mathcal{O}\left(u_{1}-u_{0}\right), \\
& \frac{\partial}{\partial w_{0}}\left(\begin{array}{c}
s_{1} \\
w_{1}
\end{array}\right)=\left(\begin{array}{c}
0_{1 \times 3} \\
1_{3 \times 3}
\end{array}\right)+\mathcal{O}\left(u_{1}-u_{0}\right) .
\end{aligned}
$$

Then the required determinant is

$$
\begin{aligned}
\mathcal{J}_{\psi}\left(u_{0}, w_{0} ; u_{0}, s_{0}\right) & =\operatorname{det}\left[-G\left(\xi_{0}, u_{0}\right),\left(\begin{array}{c}
0_{1 \times 3} \\
1_{3 \times 3}
\end{array}\right)\right] \\
& =-g_{1}\left(s_{0}, w_{0}\right),
\end{aligned}
$$

consistent with (41).

The system B2 Vlasov equation is

$$
\partial_{s} f_{2}+\nabla_{\eta} \cdot\left[H(\eta, s) f_{2}\right]=0,
$$

which is to say

$$
\partial_{s} f_{2}+H(\eta, s) \cdot \nabla_{\eta} f_{2}=-\left(\nabla_{\eta} \cdot H\right)(\eta, s) f_{2} .
$$

As a consistency check, one verifies that $f_{2}$ from (42) or (40) satisfies (47) given that $f_{1}$ satisfies (32).

\section{FINAL BEAM SYSTEM EQUATIONS OF MOTION AND PHASE SPACE DENSITY}

The B2 phase space coordinates are $\eta=\left(u, x, p_{s}, p_{x}\right)$ with evolution variable $s$. We now define the usual system B phase space coordinates by replacing $\left(u, p_{s}\right)$ with $\left(z, p_{z}\right)$, where

$$
z:=s-\beta_{r} u, \quad p_{z}:=\left[\gamma\left(p_{s}, p_{x}\right)-\gamma_{r}\right] / \gamma_{r} .
$$

We refer to $\left(z, x, p_{z}, p_{x}\right)$ as the system $\mathrm{B}$ coordinates. These are all "small" in the bunch and so linearization in the B equations of motion will be possible. Three comments about $z$ are in order. First, the $u$ density in B2 gives the distribution of arrival times at arclength $s$ and so the $z$ density in B will contain the same information. Second, a system B particle with "spatial" coordinates $(z, x)$ at arclength $s$ arrives at $s$ at time $u=(s-z) / \beta_{r}$. This particle is at system L spatial position $\mathbf{R}_{r}(s)+x \mathbf{n}(s)$ at time $u$; thus, its distance from the reference particle is $\mid \mathbf{R}_{r}(s)+x \mathbf{n}(s)-$ $\mathbf{R}_{r}\left(\beta_{r} u=s-z\right) \mid \approx \sqrt{z^{2}+x^{2}}$. Finally, a particle with $z>$ 0 arrives at arclength $s$ before the reference particle and thus is in the head of the bunch. Physically we are interested in the energy deviation of a particle from the energy $m \gamma_{r} c^{2}$ of the reference particle and $p_{z}$ is the relative energy deviation.

To determine the system B phase space density $f_{B}$ in terms of $f_{2}$, we need the Jacobian of the transformation defined by (48). The Jacobian is

$$
\frac{\partial\left(u, x, p_{s}, p_{x}\right)}{\partial\left(z, x, p_{z}, p_{x}\right)}=\frac{\partial p_{s}}{\partial p_{z}}(\mathbf{p}) \frac{1}{\beta_{r}}=\frac{1+p_{z}}{p_{s}(\mathbf{p}) \beta_{r}^{3}},
$$

with $\mathbf{p}=\left(p_{z}, p_{x}\right)$ and

$$
\begin{aligned}
p_{s}(\mathbf{p}) & =\left[\left(\frac{1}{\beta_{r}}\right)^{2}\left(1+p_{z}\right)^{2}-p_{x}^{2}-\frac{1}{\gamma_{r}^{2} \beta_{r}^{2}}\right]^{1 / 2} \\
& =\left[1+\left(2 p_{z}+p_{z}^{2}\right) / \beta_{r}^{2}-p_{x}^{2}\right]^{1 / 2} .
\end{aligned}
$$

Thus,

$$
\begin{aligned}
f_{B}\left(z, x, p_{z}, p_{x} ; s\right)= & f_{2}\left(\frac{s-z}{\beta_{r}}, x, p_{s}\left(p_{z}, p_{x}\right), p_{x} ; s\right) \\
& \times \frac{1+p_{z}}{p_{s}\left(p_{z}, p_{x}\right) \beta_{r}^{3}} .
\end{aligned}
$$

From (48), $1+p_{z}>\gamma_{r}^{-1}$, thus the Jacobian is positive.

Under the transformation, the system $B$ equations of motion become

$$
z^{\prime}=1-\frac{[1+\kappa(s) x]\left(1+p_{z}\right)}{p_{s}(\mathbf{p})}
$$

$$
x^{\prime}=\frac{[1+\kappa(s) x] p_{x}}{p_{s}(\mathbf{p})},
$$




$$
\begin{gathered}
p_{z}^{\prime}=\frac{q[1+x \kappa(s)]}{m \gamma_{r} c^{2}}\left[\mathbf{t}(s)+\frac{p_{x}}{p_{s}(\mathbf{p})} \mathbf{n}(s)\right] \\
\cdot \mathbf{E}_{\|}\left(\mathbf{T}(s, x), \frac{s-z}{\beta_{r}}\right), \\
p_{x}^{\prime}=p_{s}(\mathbf{p}) \kappa(s)-\frac{q}{P_{r}}[1+\kappa(s) x] B_{\mathrm{ext}}\left[Z_{r}(s)-x X_{r}^{\prime}(s)\right] \\
+\frac{q\left(1+p_{z}\right)}{P_{r} \beta_{r} c p_{s}(\mathbf{p})}[1+\kappa(s) x] \mathbf{n}(s) \cdot \mathbf{E}_{\|}\left(\mathbf{T}(s, x), \frac{s-z}{\beta_{r}}\right) \\
-\frac{q}{P_{r}}[1+\kappa(s) x] B_{Y}\left(\mathbf{T}(s, x), \frac{s-z}{\beta_{r}}\right) .
\end{gathered}
$$

To derive (54) differentiate $p_{z}$ from (48) and substitute $u^{\prime}$ from (38) to obtain

$$
\begin{aligned}
p_{z}^{\prime} & =\gamma^{\prime} / \gamma_{r}=\mathbf{P} \cdot \dot{\mathbf{P}} u^{\prime} / \gamma \gamma_{r} m^{2} c^{2} \\
& =\frac{q[1+\kappa(s) x]}{m \gamma_{r} c^{2}}\left[\mathbf{t}(s)+\frac{p_{x}}{p_{s}} \mathbf{n}(s)\right] \cdot \mathbf{E}_{\|} .
\end{aligned}
$$

The other equations above follow obviously from (38).

The system B phase space is

$S_{B}=\left\{\left(z, x, p_{z}, p_{x}\right):|x|<x_{M}, p_{z}>\left(\gamma_{r}^{-2}+\beta_{r}^{2} p_{x}^{2}\right)^{1 / 2}-1\right\}$.
This is the image of $S_{2}$ under the transformation (48). So the support of $f_{B}(\cdot ; s)$ is a compact subset of $S_{B}$ centered about the reference particle at $z=x=p_{z}=p_{x}=0$.

The system $B$ equations of motion (52)-(55) can be written compactly as

$$
\zeta^{\prime}=B(s, \zeta)
$$

where $\zeta=\left(z, x, p_{z}, p_{x}\right)$. Thus the Vlasov equation in $\mathrm{B}$ is

$$
\partial_{s} f_{B}+B(s, \zeta) \cdot \nabla_{\zeta} f_{B}=0
$$

where we have made use of the fact that the vector field $B(s, \cdot)$ is divergence free.

\section{RELATION BETWEEN THE L AND B DENSITIES}

We can now combine our sequence of transformations [ $f_{L} \rightarrow f_{1}$ in (24), $f_{1} \rightarrow f_{2}$ in (42) and $f_{2} \rightarrow f_{B}$ in (51)], to obtain

$$
f_{B}\left(z, x, p_{z}, p_{x} ; s\right)=\frac{P_{r}^{2}}{\beta_{r}^{2}} f_{L}\left(\mathbf{R}(s)+x \mathbf{n}(s), P_{r}\left[p_{s}(\mathbf{p}) \mathbf{t}(s)+p_{x} \mathbf{n}(s)\right] ; \frac{s-z}{\beta_{r}}\right) .
$$

Equivalently, the transformation in the other order gives

$$
f_{L}(\mathbf{R}, \mathbf{P} ; u)=\frac{\beta_{r}^{2}}{P_{r}^{2}} f_{B}\left[\hat{s}(\mathbf{R})-\beta_{r} u, \hat{x}(\mathbf{R}), \hat{p}_{z}(\mathbf{P}), \hat{p}_{x}(\mathbf{R}, \mathbf{P}) ; \hat{s}(\mathbf{R})\right] .
$$

To recapitulate, the coordinate transformations in extended phase space are written as

$$
\begin{gathered}
\mathbf{R}=\mathbf{R}_{r}(s)+x \mathbf{n}(s), \quad \mathbf{P}=P_{r}\left[p_{s}(\mathbf{p}) \mathbf{t}(s)+p_{x} \mathbf{n}(s)\right], \\
u=(s-z) / \beta_{r},
\end{gathered}
$$

with inverse

$$
\begin{aligned}
s & =\hat{s}(\mathbf{R}), \quad z=\hat{s}(\mathbf{R})-\beta_{r} u, \quad x=\hat{x}(\mathbf{R}), \\
p_{z} & =\hat{p}_{z}(\mathbf{P}):=-1+\left(1+\mathbf{P} \cdot \mathbf{P} / m^{2} c^{2}\right)^{1 / 2} / \gamma_{r}, \\
p_{x} & =\hat{p}_{x}(\mathbf{R}, \mathbf{P}):=\mathbf{P} \cdot \mathbf{n}[\hat{s}(\mathbf{R})] / P_{r} .
\end{aligned}
$$

To determine the charge density in terms of the system $B$ phase space density we use (5) and (59) to obtain

$$
\begin{aligned}
\rho_{L}(\mathbf{R} ; u)= & Q \int f_{L}(\mathbf{R}, \mathbf{P} ; u) d \mathbf{P} \\
= & Q \int \frac{\left(1+p_{z}\right)}{p_{s}(\mathbf{p})} f_{B}[\hat{s}(\mathbf{R}) \\
& \left.-\beta_{r} u, \hat{x}(\mathbf{R}), \mathbf{p} ; \hat{s}(\mathbf{R})\right] d \mathbf{p} .
\end{aligned}
$$

To determine the current density in terms of the B phase space density we use (6) and (59) to obtain

$$
\begin{aligned}
\mathbf{J}_{L}(\mathbf{R} ; u)= & Q \int \frac{\mathbf{P}}{m \gamma(\mathbf{P})} f_{L}(\mathbf{R}, \mathbf{P} ; u) d \mathbf{P} \\
= & Q \beta_{r} c \int\left[\mathbf{t}[\hat{s}(\mathbf{R})]+\frac{p_{x}}{p_{s}(\mathbf{p})} \mathbf{n}[\hat{s}(\mathbf{R})]\right] \\
& \cdot f_{B}\left[\hat{s}(\mathbf{R})-\beta_{r} u, \hat{x}(\mathbf{R}), \mathbf{p} ; \hat{s}(\mathbf{R})\right] d \mathbf{p} .
\end{aligned}
$$

The formulas (62) and (63) are derived by substituting (59) and changing the variable of integration from $\mathbf{P}$ to $\mathbf{p}$. The Jacobian of the transformation is

$$
\begin{aligned}
\operatorname{det}\left[\frac{\partial \mathbf{P}}{\partial p_{z}}, \frac{\partial \mathbf{P}}{\partial p_{x}}\right] & =P_{r}^{2} \operatorname{det}\left[\frac{\partial p_{s}}{\partial p_{z}} \mathbf{t}, \frac{\partial p_{s}}{\partial p_{x}} \mathbf{t}+\mathbf{n}\right] \\
& =P_{r}^{2} \operatorname{det}\left[\frac{\partial p_{s}}{\partial p_{z}} \mathbf{t}, \mathbf{n}\right]=P_{r}^{2} \frac{\partial p_{s}}{\partial p_{z}} \\
& =\frac{P_{r}^{2}}{\beta_{r}^{2}} \frac{1+p_{z}}{p_{s}(\mathbf{p})} .
\end{aligned}
$$

Even though the derivation of (58) and (59) is somewhat subtle and a bit complex, the end result is quite simple. 


\section{REMARK ON THE SYSTEM B VLASOV EQUATION}

The beam system Vlasov equation (57) is to be solved as an initial value problem. In practice this is done numerically in small steps $\Delta s$ of the independent variable, with the collective force held constant as a function of $s$ during an integration step. We shall now show that the initial value problem is in fact not well posed: a knowledge of $f_{B}(\cdot ; \tau)$ for $\tau \leq s$ is not sufficient, strictly speaking, to find the collective force required to compute $f_{B}(\cdot ; s+\Delta s)$. In addition, one requires $f_{B}(\cdot, \tau)$ for $\tau$ slightly larger than $s$, say for $s<\tau<s+\Delta$. This apparent "failure of causality" is not a bother in computations, since one needs anyway to treat $f_{B}$ as constant over a step $\Delta s$ that is typically much larger than $\Delta$. For a perfectly rigorous treatment it appears that the $\mathrm{B} 2$ and $\mathrm{B}$ descriptions are simply not suited to the initial value problem.

Suppose that $f_{B}(\cdot ; \tau)$ is known for $\tau \leq s$. To integrate the Vlasov equation we have to follow characteristics, the single-particle orbits determined by (52)-(55). In (54) and (55) we have the collective force $\mathcal{F}_{L}\left[\mathbf{T}(s, x),(s-z) / \beta_{r}\right]$ and thus from (3) we need $\mathbf{S}\left[\mathbf{R}^{\prime},(s-z) / \beta_{r}-\mid \mathbf{R}^{\prime}-\right.$ $\mathbf{T}(s, x) \mid]$ for all $\mathbf{R}^{\prime} \in \mathbb{R}^{2}$. The latter requires $\rho_{L}\left[\mathbf{R}^{\prime} ;(s-\right.$ $\left.z) / \beta_{r}-\left|\mathbf{R}^{\prime}-\mathbf{T}(s, x)\right|\right]$ which from (62) involves

$$
f_{B}\left[\hat{s}\left(\mathbf{R}^{\prime}\right)-s+z+\beta_{r}\left|\mathbf{R}^{\prime}-\mathbf{T}(s, x)\right|, \hat{x}\left(\mathbf{R}^{\prime}\right), \mathbf{p} ; \hat{s}\left(\mathbf{R}^{\prime}\right)\right] .
$$

Now consider $z=x=0$, which is certainly a point at which the collective force must be known. Also consider a value of the integration variable $\mathbf{R}^{\prime}=\mathbf{R}_{r}(s)+\delta \mathbf{t}(s)$, where $\delta$ is positive and small in the sense that $|\kappa \delta| \ll 1$. For this $\mathbf{R}^{\prime}$, taking $y=s$ in (21) gives $\hat{s}\left(\mathbf{R}^{\prime}\right)=s+\delta+$ $\mathcal{O}\left(\kappa \delta^{2}\right)$ and $\hat{x}\left(\mathbf{R}^{\prime}\right)=\mathcal{O}\left(\kappa \delta^{2}\right)$. So finally by (65) we need $f_{B}\left[\left(1+\beta_{r}\right) \delta, \mathcal{O}\left(\kappa \delta^{2}\right), \mathbf{p} ; s+\delta\right]$. The density arguments are clearly within the bunch support for small $\mathbf{p}$ and this completes the claim: the density $f_{B}(\cdot ; s+\delta)$ is involved in the collective force at $s$.

A geometrical discussion of this "causality issue" is given in $[7,8]$.

\section{APPROXIMATIONS}

A. Approximate relation between $\rho_{L}, \mathrm{~J}_{L}$, and $f_{B}$

A Taylor expansion based on (50) gives

$$
\begin{aligned}
\left(1+p_{z}\right) / p_{s}(\mathbf{p}) & =1+\left(\gamma_{r}\right)^{-2} p_{z}+\mathcal{O}\left(p_{z}^{2}, p_{x}^{2}\right), \\
p_{x} / p_{s}(\mathbf{p}) & =p_{x}+\mathcal{O}\left(p_{x} p_{z}, p_{x}^{3}\right) .
\end{aligned}
$$

So to good approximation, (62) and (63) yield

$$
\begin{gathered}
\rho_{L}(\mathbf{R} ; u) \approx \rho_{B}\left[\hat{s}(\mathbf{R})-\beta_{r} u, \hat{x}(\mathbf{R}) ; \hat{s}(\mathbf{R})\right], \\
\mathbf{J}_{L}(\mathbf{R} ; u) \approx \beta_{r} c\left\{\rho_{B}\left[\hat{s}(\mathbf{R})-\beta_{r} u, \hat{x}(\mathbf{R}) ; \hat{s}(\mathbf{R})\right] \mathbf{t}[\hat{s}(\mathbf{R})]\right. \\
\left.+\tau_{B}\left[\hat{s}(\mathbf{R})-\beta_{r} u, \hat{x}(\mathbf{R}) ; \hat{s}(\mathbf{R})\right] \mathbf{n}[\hat{s}(\mathbf{R})]\right\},
\end{gathered}
$$

where the B charge density is

$$
\rho_{B}(z, x ; s):=Q \int f_{B}\left(z, x, p_{z}, p_{x} ; s\right) d p_{z} d p_{x},
$$

and the "transverse component" of the current involves

$$
\tau_{B}(z, x ; s):=Q \int p_{x} f_{B}\left(z, x, p_{z}, p_{x} ; s\right) d p_{z} d p_{x} .
$$

The support of $\rho_{L}(\mathbf{R} ; u)$ is in a small region about $\mathbf{R}=$ $\mathbf{R}_{r}\left(\beta_{r} u\right)$. For this region we take $y=\beta_{r} u$ in (21) to obtain

$$
\begin{aligned}
\left(\begin{array}{c}
\hat{s}(\mathbf{R})-\beta_{r} u \\
\hat{x}(\mathbf{R})
\end{array}\right)= & M^{T}\left(\beta_{r} u\right)\left[\mathbf{R}-\mathbf{R}_{r}\left(\beta_{r} u\right)\right] \\
& +\mathcal{O}\left[\kappa\left\|\mathbf{R}-\mathbf{R}_{r}\left(\beta_{r} u\right)\right\|^{2}\right] .
\end{aligned}
$$

To a very good approximation the error term can be ignored because for $\mathbf{R}$ in the support of $\rho_{L}(\mathbf{R} ; u),|\kappa| \| \mathbf{R}-$ $\mathbf{R}_{r}\left(\beta_{r} u\right) \| \ll 1$. Thus in the support of $\rho_{L}$, we have

$$
\begin{aligned}
\rho_{L}(\mathbf{R} ; u) \approx & \rho_{B}\left\{M^{T}\left(\beta_{r} u\right)\left[\mathbf{R}-\mathbf{R}_{r}\left(\beta_{r} u\right)\right] ;\right. \\
& \left.\beta_{r} u+\mathbf{t}\left(\beta_{r} u\right)^{T}\left[\mathbf{R}-\mathbf{R}_{r}\left(\beta_{r} u\right)\right]\right\} .
\end{aligned}
$$

Now we make the usually acceptable approximation that the beam frame bunch form changes very little when $s$ varies by an amount comparable to the bunch size. That is, $f_{B}(\cdot ; s+\Delta) \approx f_{B}(\cdot ; s)$ for $|\Delta|$ less than the $z$ size of the beam. Then we can drop the last term in the final argument of $\rho_{B}$. With similar approximations for the current density we then have the final formulas suitable for applications:

$$
\begin{aligned}
\rho_{L}(\mathbf{R} ; u) \approx \rho_{B}\left\{M^{T}\left(\beta_{r} u\right)\left[\mathbf{R}-\mathbf{R}_{r}\left(\beta_{r} u\right)\right] ; \beta_{r} u\right\}, \\
\mathbf{J}_{L}(\mathbf{R} ; u) \approx \beta_{r} c\left(\rho_{B}\left\{M^{T}\left(\beta_{r} u\right)\left[\mathbf{R}-\mathbf{R}_{r}\left(\beta_{r} u\right)\right] ; \beta_{r} u\right\} \mathbf{t}\left(\beta_{r} u\right)\right. \\
\left.+\tau_{B}\left\{M^{T}\left(\beta_{r} u\right)\left[\mathbf{R}-\mathbf{R}_{r}\left(\beta_{r} u\right)\right] ; \beta_{r} u\right\} \mathbf{n}\left(\beta_{r} u\right)\right) .
\end{aligned}
$$

These are the beam frame to lab frame density relations we have used in certain of our numerical computations.

\section{B. Approximate equations of motion in system B}

Here we derive equations of motion that approximate (56) for small $\zeta$. The components of $B=\left(b_{1}, b_{2}, b_{3}, b_{4}\right)$ are defined by (52)-(55). We wish to linearize the terms which do not involve self-fields, neglecting terms quadratic and higher in the components of $\zeta$, thereby obtaining formulas which are familiar in the literature and useful in computations. We shall also linearize coefficients of the self-fields $\mathbf{E}_{\|}$and $B_{Y}$, to a consistent order of approximation.

From (66) we obtain

$$
\begin{aligned}
(1+\kappa x)\left(1+p_{z}\right) / p_{s}(\mathbf{p})= & 1+\kappa x+\gamma_{r}^{-2} p_{z} \\
& +\mathcal{O}\left(\gamma_{r}^{-2} \kappa x p_{z}, p_{z}^{2}, p_{x}^{2}\right) \\
(1+\kappa x) p_{x} / p_{s}(\mathbf{p})=p_{x} & +\mathcal{O}\left(\kappa x p_{x}, p_{x} p_{z}^{2}, p_{x}^{3}\right)
\end{aligned}
$$


Applying these and dropping nonlinear terms, we obtain $b_{1}=-\kappa x-\gamma_{r}^{-2} p_{z}, b_{2}=p_{x}$, and $b_{3}=\left(q / m \gamma_{r} c^{2}\right)[(1+$ $\left.\kappa x) \mathbf{t}+p_{x} \mathbf{n}\right] \cdot \mathbf{E}_{\|}$. Neglecting $\kappa(s) x$ in comparison to 1 , because the transverse bunch size is very small compared to the bending radius, we obtain $b_{3}=\left(q / m \gamma_{r} c^{2}\right) \times$ $\left(\mathbf{t}+p_{x} \mathbf{n}\right) \cdot \mathbf{E}_{\|}$. We neglect $\kappa x$ in the fourth term of $b_{4}$ for the same reason and using (75) the third term in $b_{4}$ becomes $\left(q / P_{r} \beta_{r} c\right) \mathbf{n} \cdot \mathbf{E}_{\|}$where we also neglect $\gamma_{r}^{-2} p_{z}$ in comparison with 1 . The first two terms in $b_{4}$ can be written $\beta_{r}^{-2} \kappa p_{z}+\mathcal{E}(s, x)+\mathcal{O}\left(\kappa p_{z}^{2}, \kappa p_{x}^{2}\right)$, where

$$
\begin{aligned}
\mathcal{E}(s, x) & =\frac{q}{P_{r}}\left[B_{\mathrm{ext}}\left(Z_{r}\right)-(1+\kappa x) B_{\mathrm{ext}}\left(Z_{r}-x X_{r}^{\prime}\right)\right] \\
& =-\kappa^{2} x+\frac{q}{P_{r}}(1+\kappa x)\left[B_{\mathrm{ext}}\left(Z_{r}\right)-B_{\mathrm{ext}}\left(Z_{r}-x X_{r}^{\prime}\right)\right]
\end{aligned}
$$

is the so-called edge effect term. Thus to good approximation

$$
b_{4}=\beta_{r}^{-2} \kappa p_{z}+\mathcal{E}(s, x)+\frac{q}{P_{r} \beta_{r} c}\left[\mathbf{n}(s) \cdot \mathbf{E}_{\|}-\beta_{r} c B_{Y}\right] .
$$

In our work $\gamma_{r}$ is large and we neglect $p_{z} / \gamma_{r}^{2}$ in $b_{1}$ and replace $\beta_{r}$ by 1 in $b_{4}$. Our approximate equations of motion are

$$
\begin{aligned}
z^{\prime} & =-\kappa(s) x, \quad x^{\prime}=p_{x}, \\
p_{z}^{\prime} & =\frac{q}{P_{r} c}\left[\mathbf{t}(s)+p_{x} \mathbf{n}(s)\right] \cdot \mathbf{E}_{\|}, \\
p_{x}^{\prime} & =\kappa(s) p_{z}+\frac{q}{P_{r} c}\left[\mathbf{n}(s) \cdot \mathbf{E}_{\|}-c B_{Y}\right]+\mathcal{E}(s, x),
\end{aligned}
$$

where the self-fields $\mathbf{E}_{\|}, B_{Y}$ are evaluated at $(\mathbf{R}, u)=$ $\left[\mathbf{T}(s, x),(s-z) / \beta_{r}\right]$ with $\mathbf{T}(s, x)=R_{r}(s)+x \mathbf{n}(s)$. These equations (apart from the self-field and edge effect terms) are standard in the literature [9]. We have applied (78), with $\mathcal{E}=0$, in a series of works, using Eqs. (73) and (74) to relate $\rho_{L}, \mathbf{J}_{L}$ and $\rho_{B}, \tau_{B}$; see Refs. [1,3-5,7].

We end this section with a brief discussion of the edge effect term. To good approximation the $\kappa x$ term can be dropped in the second expression for $\mathcal{E}$ in (77) (but not in the first). We write $\mathcal{E}=\mathcal{E}_{f}+\mathcal{E}_{d f}$, where $\mathcal{E}_{f}(s, x)=$ $-\kappa(s)^{2} x$ and

$$
\mathcal{E}_{d f}(s, x)=\frac{q}{P_{r}}\left[B_{\text {ext }}\left(Z_{r}\right)-B_{\text {ext }}\left(Z_{r}-x X_{r}^{\prime}\right)\right] .
$$

In our applications we have only hard edge dipole magnets and drift spaces. In the drift spaces $\mathcal{E}=0$. In the magnets $\mathcal{E}=\mathcal{E}_{f}$ which gives rise to a focusing effect. It turns out that this focusing effect is counterbalanced, to some extent, by $\mathcal{E}_{d f}$, which is only nonzero near the edge of a magnet and acts to defocus the bunch. For small bending angle, the effect of $\mathcal{E}_{d f}$ can be approximated by a kick in $p_{x}$, as is well known by, e.g., users of MAD [10]. In conclusion, we have found, in our applications, that approximating $\mathcal{E}$ with either $\mathcal{E}_{f}$ or $\mathcal{E}_{d f}$ introduces an unphysical, non-negligible focus- ing/defocusing effect, while neglecting $\mathcal{E}$ has a small effect on $p_{x}$ which can be ignored.

\section{ACKNOWLEDGMENTS}

This work has been supported by DOE under AC0376SF00515 and DE-FG02-99ER41104.

\section{APPENDIX: JACOBIAN DETERMINANT FOR A TRANSFORMATION OF DENSITIES}

Here we prove formula (41) for the Jacobian $\mathcal{J}_{\psi}$. The operator $D_{i}$ denotes partial differentiation with respect to the $i$ th argument of the function on which it operates; an argument may be scalar or vector. In this notation the function $\varphi$ of (31) [see also (39)] satisfies the initial value problem,

$$
\begin{gathered}
D_{1} \varphi\left(u_{1}, u_{0} \mid \xi_{0}\right)=G\left[\varphi\left(u_{1}, u_{0} \mid \xi_{0}\right), u_{1}\right], \\
\varphi\left(u_{0}, u_{0} \mid \xi_{0}\right)=\xi_{0} .
\end{gathered}
$$

Here $\xi_{0}=\left(s_{0}, w_{0}\right)$ and $G$ is defined by (30), (33), and (34). Differentiating (A1) with respect to $\xi_{0}$ we obtain the linear variational equation satisfied by the $4 \times 4$ matrix $\Phi\left(u_{1}, u_{0} \mid \xi_{0}\right):=D_{3} \varphi\left(u_{1}, u_{0} \mid \xi_{0}\right):$

$$
D_{1} \Phi\left(u_{1}, u_{0} \mid \xi_{0}\right)=D_{1} G\left[\varphi\left(u_{1}, u_{0} \mid \xi_{0}\right), u_{1}\right] \Phi\left(u_{1}, u_{0} \mid \xi_{0}\right)
$$

$$
\Phi\left(u_{0}, u_{0} \mid \xi_{0}\right)=I_{4 \times 4} .
$$

For the required Jacobian we need $D_{2} \varphi$. By differentiating (A1) with respect to $u_{0}$ and then changing the order of derivatives, we see that this function is a vector solution of the same variational equation:

$$
\begin{gathered}
D_{1}\left[D_{2} \varphi\left(u_{1}, u_{0} \mid \xi_{0}\right)\right]=D_{1} G\left[\varphi\left(u_{1}, u_{0} \mid \xi_{0}\right), u_{1}\right] \\
\times\left[D_{2} \varphi\left(u_{1}, u_{0} \mid \xi_{0}\right)\right], \\
D_{2} \varphi\left(u_{0}, u_{0} \mid \xi_{0}\right)=-G\left(\xi_{0}, u_{0}\right) .
\end{gathered}
$$

The initial value was found by differentiating the identity $\varphi\left(u_{0}, u_{0} \mid \xi_{0}\right)=\xi_{0}$ and applying (A1). Since $\Phi$ is the principal solution matrix of the linear variational equation, $D_{2} \varphi$ must be just a linear combination of the columns of $\Phi$, namely, the combination that has the right initial value. In view of (A6) and (A4) that is

$$
D_{2} \varphi\left(u_{1}, u_{0} \mid \xi_{0}\right)=-\Phi\left(u_{1}, u_{0} \mid \xi_{0}\right) G\left(\xi_{0}, u_{0}\right) .
$$

Similarly, the $4 \times 3$ matrix $D_{4} \varphi\left(u_{1}, u_{0} \mid s_{0}, w_{0}\right)$, again a solution of the variational equation, is represented as

$$
D_{4} \varphi\left(u_{1}, u_{0} \mid s_{0}, w_{0}\right)=\Phi\left(u_{1}, u_{0} \mid \xi_{0}\right)\left(\begin{array}{c}
0_{1 \times 3} \\
I_{3 \times 3}
\end{array}\right) .
$$

The required determinant in (41) is now easily evaluated from (A7) and (A8) as 


$$
\begin{aligned}
& \operatorname{det}\left[D_{1} \psi, D_{2} \psi\right] \\
& =\operatorname{det}\left[D_{2} \varphi, D_{4} \varphi\right] \\
& =\operatorname{det} \Phi\left(u_{1}, u_{0} \mid \xi_{0}\right) \operatorname{det}\left[-G\left(\xi_{0}, u_{0}\right),\left(\begin{array}{c}
0_{1 \times 3} \\
I_{3 \times 3}
\end{array}\right)\right] \\
& =-g_{1}\left(\xi_{0}\right) \exp \int_{u_{0}}^{u_{1}} \operatorname{Tr} D_{1} G\left[\varphi\left(u, u_{0} \mid \xi_{0}\right), u\right] d u .
\end{aligned}
$$

The exponential is the Abel-Liouville expression [11] for $\operatorname{det} \Phi\left(u_{1}, u_{0} \mid \xi_{0}\right)$.

[1] G. Bassi, J. A. Ellison, K. Heinemann, and R. Warnock, Phys. Rev. ST Accel. Beams 12, 080704 (2009).

[2] É. Forest, Beam Dynamics: a New Attitude and Framework (CRC Press, Atlanta, 1998).

[3] R. Warnock, G. Bassi, and J. A. Ellison, Nucl. Instrum. Methods Phys. Res., Sect. A 558, 85 (2006).

[4] G. Bassi, J. A. Ellison, K. Heinemann, M. Venturini, and R. Warnock, in Proceedings of the 2007 Particle Accelerator Conference, Albuquerque, New Mexico
(IEEE, Albuquerque, New Mexico, 2007), paper TUZBC03.

[5] G. Bassi, J. A. Ellison, K. Heinemann, and R. Warnock, in Proceedings of the 2007 Particle Accelerator Conference, Albuquerque, New Mexico (Ref. [4]), paper THPAN084.

[6] G. Bassi, J. A. Ellison, K. Heinemann, and R. Warnock, in Proceedings of the 23rd Particle Accelerator Conference, Vancouver, Canada, 2009 (IEEE, Piscataway, NJ, 2009), paper TU1PBI03.

[7] G. Bassi, J. A. Ellison, and K. Heinemann, in Proceedings of ICAP09, San Francisco, CA, USA, paper TH2IOpk01.

[8] K. Heinemann, Ph.D. dissertation, University of New Mexico, 2010.

[9] S. Heifets, G. Stupakov, and S. Krinsky, Phys. Rev. ST Accel. Beams 5, 064401 (2002); 5, 129902(E) (2002); H. Huang and K. Kim, Phys. Rev. ST Accel. Beams 5, 074401 (2002); 5, 129903(E) (2002).

[10] See http://mad.web.cern.ch/mad/.

[11] P. Hartman, Ordinary Differential Equations (Wiley, New York, 1964), p. 64; C. Chicone, Ordinary Differential Equations with Applications (Springer, Berlin, 1999), p. 134. 